\title{
Mutation analysis of the lamin A/C gene (LMNA) among patients with different cardiomuscular phenotypes
}

\author{
M Vytopil, S Benedetti, E Ricci, G Galluzzi, A Dello Russo, L Merlini, G Boriani, M Gallina, \\ L Morandi, L Politano, M Moggio, L Chiveri, I Hausmanova-Petrusewicz, R Ricotti, S Vohanka, \\ J Toman, D Toniolo
}

L aminopathies represent a heterogeneous group of genetic disorders characterised by mutations in the LMNA gene, -which encodes two lamins, A and C, by alternative splicing of the primary transcript. ${ }^{1}$ Lamins belong to the intermediate filament multigene family and form the nuclear lamina, a mesh-like structure adjacent to the nucleoplasmic side of the inner nuclear membrane. ${ }^{2}$ They interact with emerin, the proteins encoded by the gene for the X-linked ( $\mathrm{X}$ EDMD) form of EDMD, with several nuclear envelope proteins and with chromatin. Despite their widespread distribution and their role in nuclear architecture, alterations of lamin $\mathrm{A} / \mathrm{C}$ are responsible for a number of very specific but quite heterogeneous disorders.

The first laminopathy was the autosomal dominant form of Emery-Dreifuss muscular dystrophy (EDMD), a genetic disorder characterised by the clinical triad of early onset contractures, progressive muscular wasting and weakness with humeroperoneal distribution and cardiac conduction defects. ${ }^{3}$ The finding that emerin, an inner nuclear envelope protein, and LMNA were both involved in EDMD suggested that the lamins may represent specific and relevant factors in cardiac and skeletal muscle and that integrity of the nuclear membrane and associated structures is specifically required for muscle function.

However, later on it was found that besides autosomal dominant Emery-Dreifuss muscular dystrophy (AD-EDMD), mutations in LMNA are responsible for six other disorders: limb girdle muscular dystrophy 1B, (LGMD1B), ${ }^{45}$ dilated cardiomyopathy with conduction system disease, (DCMCD), ${ }^{6}$ Dunningan-type familial partial lipodystrophy, ${ }^{7-9}$ one recessive axonal form of Charcot-Marie-Tooth neuropathy, ${ }^{10}$ mandibuloacral dysplasia, ${ }^{11}$ and Hutchinson Gilford progeria. $^{12} 13$

Despite the very different phenotypic consequences of mutations in $L M N A$, and the quite large number of mutations identified, no genotype/phenotype correlation has been demonstrated, pointing to the role of factors other than lamins $\mathrm{A}$ and $\mathrm{C}$ in determining the different tissue specific phenotypes. Mutation analysis of the LMNA gene has shown that mutations are predominantly missense and do not cause a reduction in the amount of lamin $\mathrm{A} / \mathrm{C}$ at the nuclear lamina. However these mutations must lead to perturbances of the nuclear lamina responsible for the phenotype through alterations of interactions with emerin and with other proteins that can confer the tissue specificity of the disorders.

Three of the autosomal dominant disorders affect skeletal and cardiac muscle to a various degree. LGMDIB (MIM $159001)$ belongs to a heterogeneous group of muscular dystrophies. ${ }^{5}$ It is inherited as an autosomal dominant trait, characterised by slowly progressive limb girdle weakness and wasting, atrioventricular cardiac conduction defects and dilated cardiomyopathy. Patients affected with LGMDIB differ from patients with $\mathrm{AD}-\mathrm{EDMD}$ as they lack early

\section{Key points}

- The aim of the work was to study the frequency of mutations in the LMNA gene associated to the different cardiac and skeletal muscle phenotypes described and that seem to form a group of overlapping entities with marked clinical variability.

- We collected 166 patients who could be divided into four groups: Emery Dreifuss muscular dystrophy (EDMD), isolated heart disease, isolated muscular dystrophy, and idiopathic hyperCKaemia. All patients were positive for emerin immunohistochemistry.

- Mutation analysis of LMNA demonstrates that the presence of heart involvement and age of onset are distinguishing features separating patients with EDMD and LMNA mutation from patients with EDMD who are LMNA/STA negative, while distribution of myopathy is not a reliable diagnostic criterion in EDMD.

- The frequency of LMNA mutations in isolated heart disease is very low (3-5\%) but similar to that of other genes identified, underscoring the high heterogeneity of the cardiac phenotype and a role of LMNA in this group of disorders.

- Patients carrying a wild type LMNA gene did not carry missense mutations in the X-linked gene STA.

- At least one other gene will have to be identified to characterise the EDMD phenotype fully.

contractures and show predominant proximal myopathy. The heart involvement in DCM-CD (MIM 115200) is clinically similar to the cardiac disease in AD-EDMD and LGMD IB and takes the form of conduction system disease and dilated cardiomyopathy. ${ }^{6}$ Patients do not present with contractures or muscle wasting or weakness. The overlapping features of these disorders suggest that the three autosomal dominant disorders affecting skeletal and cardiac muscle may represent one disease entity with variable expression of symptoms and that the boundaries between the three phenotypes are unclear. At least in one case, a mutation in LMNA gene was shown to account for all three phenotypes within a single family. ${ }^{14}$ Among the LMNA disorders, dilated cardiomyopathy is the disease of highest sociomedical importance since it

Abbreviations: $A D-E D M D$, autosomal dominant Emery-Dreifuss muscular dystrophy; $A V$, atrioventricular; $C K$, creatine kinase; $D C M-C D$, dilated cardiomyopathy with conduction system disease; EDMD, EmeryDreifuss muscular dystrophy; LGMD1B, limb girdle muscular dystrophy 1B; X EDMD, X-linked Emery-Dreifuss muscular dystrophy 
Table 1 Groups of patients analysed

\begin{tabular}{llll}
\hline Clinical syndromes & Clinical subgroups & Number of patients & Number of mutations (\%) \\
\hline $\mathbf{1}$ EDMD & & 38 & $15(39.5)$ \\
$\mathbf{2}$ Isolated heart disorders & dilated cardiomyopathy & 30 & $1(3.3)$ \\
& conduction defects, arrhythmia & 25 & $1(4)$ \\
3 Myopathy & combination & 16 & none \\
& with heart involvement & 20 & 12 \\
$\mathbf{4}$ Idiopathic hyperCKaemia & without heart involvement & 23 & none \\
5 Lipodystrophy & & 2 & none \\
\hline
\end{tabular}

occurs with high population frequency, ${ }^{15}$ many affected individuals are young and they frequently face dismal prognosis. To reach a more exact estimation of the frequency of LMNA mutations in different laminopathies and to try to refine the clinical and genetic boundaries between individual phenotypes, a large, clinically heterogeneous set of patients was analysed, and here we report the results of the mutation analysis of LMNA and emerin genes in this group of patients.

\section{PATIENTS}

One hundred and sixty six patients belonging to five clinical subgroups were collected for the analysis (table 1).

Thirty eight patients were diagnosed with EDMD based on the following criteria: (a) contractures of elbows or ankles, or rigid spine; (b) progressive muscular dystrophy with humeroperoneal, proximal, axial, or generalised distribution and onset of muscular symptoms before age 20. Patients with the muscle phenotype but not presenting with cardiac disorder after the age of 20 were also included in this group.

Seventy one unrelated patients with isolated heart disease were divided into three clinical subgroups as shown in table 1. The following inclusion criteria were applied: (a) DCM with ejection fraction less than $45 \%$ and no limit in age of onset, (b) presence of permanent or recurrent supraventricular tachyarrhythmias before age 45, (c) presence of atrioventricular conduction defects requiring insertion of pacemaker before age 45 . Patients with diabetes mellitus, atherosclerosis, heart disease, or alcoholism were excluded.

Thirty two patients with diagnostically unclassified muscular dystrophies were analysed. Twelve of them had heart disease and twenty had isolated muscular dystrophy.

Twenty three patients aged between 3 and 70 and presenting with idiopathic hyperCKaemia were also screened for mutations. Creatine kinase (CK) levels ranged from 246 U/l to $>9000 \mathrm{U} / \mathrm{l}$. Patients were all asymptomatic. The most frequent causes of muscle disorder-dystrophin, dysferlin, sarcoglycans and laminin-were excluded by analysing muscle biopsies. ${ }^{16}$

Two patients with lipodystrophy were analysed. One patient presented with partial lipodystrophy of forearms and hands and contractures of elbows. The second patient was described as having generalised Berardinelli-type lipodystrophy, hyperCKaemia, weakness of bilateral peroneals, scoliosis and arrhythmia. His parents were first degree cousins.

\section{METHODS}

\section{Genetic analysis of LMNA and STA genes}

All exons and exon-intron junctions of the LMNA and STA genes were amplified by PCR and analysed by DHPLC (Transgenomic) as described previously. ${ }^{17}$ To analyse putative homozygote mutations, the PCR products from probands were mixed with equimolar amounts of PCR products from a normal individual. The PCR products with peak profiles different from the wild type were sequenced directly using a
Perkin Elmer 373A Automated Sequencer. Primer, PCR and DHPLC conditions are listed at www.sanraffaele.org/ research/toniolo/

\section{RESULTS}

The LMNA gene was screened for mutations in a set of 166 patients presenting with EDMD or the isolated cardiac or muscular phenotype. Three patients whose parents were first degree cousins were analysed for homozygous mutations in the LMNA gene. No recessive mutations were found.

\section{EDMD-like phenotypes}

Fifteen mutations (39.5\%) were identified in 38 unrelated patients clinically defined as AD-EDMD, based on their muscle phenotype and presence of normal emerin (table 2). Twenty one patients who did not have a mutation in the $L M N A$ gene (table 3) were screened for mutations in the coding sequence of the X-linked gene STA gene. No mutations were detected in the X-linked gene.

Eleven different mutations were identified. The mutations R453W and R249Q, repeatedly reported, ${ }^{18-20}$ were found three times and twice respectively among the group of patients. A third mutation $\mathrm{n}$ (T528R) changes a $\mathrm{T}$ residue previously reported in a different mutation. ${ }^{18}$ It was found twice. Finally, the El12del mutation was also previously reported. ${ }^{18}$ The remaining seven mutations were novel. One mutation was found in the portion of first exon encoding the $\mathrm{N}$-terminal head. Six were found within LMNA exons encoding the central rod domain. Four mutations altered amino acid residues in the $\mathrm{C}$ terminal tail.

One mutation (patient CM) was a heterozygous 103C $>$ G substitution, causing the $\mathrm{L} 35 \mathrm{~V}$ amino acid change, not found among 200 chromosomes of control subjects. The nucleotide change results in a conservative substitution. However, L to V substitutions were previously shown to cause phenotypic changes and structural alterations ${ }^{21} 22$ and may possibly change the structure and the interactions of lamin $\mathrm{A} / \mathrm{C}$ as well. Patient CM had multiple contractures (elbows, knees, ankles) with onset in early childhood, spine rigidity, diffuse muscular wasting and serious supraventricular tachyarrhythmias at age 9. Healthy parents did not carry the mutation.

Patient AAg (described in greater detail elsewhere ${ }^{17}$ ) is worth mentioning here. She carried a three nucleotide deletion (94-96delAAG, K32del) and presented with severe EDMD at age 16. The mutation was inherited from her father who at age 52 was healthy. The presence of incomplete penetrance of dominant heterozygous mutations in LMNA gene was repeatedly reported ${ }^{17} 20$ and suggests that healthy parents of sporadic cases should be tested since they may be asymptomatic carriers possibly at high risk of sudden death.

\section{Isolated cardiac disease}

Two novel mutations ( $1303 \mathrm{C}>\mathrm{T}$ in patient JiR, and 1039G $>$ A in patient NE5) were identified among 71 patients with isolated cardiac disease (table 1). Thirty patients had dilated 
Table 2 Patients with EDMD cases and LMNA mutations

\begin{tabular}{|c|c|c|c|c|c|c|c|c|}
\hline & Patient & Mutation & $\begin{array}{l}\text { Protein } \\
\text { change }\end{array}$ & Age & I & $\begin{array}{l}\text { Distribution of } \\
\text { clinical myopathy }\end{array}$ & Contractures & $\begin{array}{l}\text { Cardiac } \\
\text { involvement }\end{array}$ \\
\hline 1 & SS & $73 C>G$ & R26G & 36 & nk & proximal, axial & elbow, ankle & $C D$ \\
\hline 2 & $\mathrm{AAg}$ & 94-96del AAG & K32del & 16 & fam & proximal, axial & elbow, ankle & A, DCM \\
\hline 3 & $\mathrm{CM}$ & $103 C>G$ & L35V & 9 & spor & generalised & elbow, knee, ankle, rigid spine & A \\
\hline 4 & $\mathrm{CaA}$ & 334-336 delGAG & E112del & 15 & $\mathrm{nk}$ & proximal & elbow, ankle, rigid spine & none \\
\hline 5 & SD & $743 \mathrm{~T}>\mathrm{C}$ & L248P & 15 & fam & $\begin{array}{l}\text { humeroperoneal, } \\
\text { proximal in lower limbs }\end{array}$ & elbow & none \\
\hline 6 & MLG & $746 \mathrm{G}>\mathrm{A}$ & R249Q & 28 & spor & generalised & elbow, knee, ankle, rigid spine & $A, C D, P M$ \\
\hline 7 & And & $746 G>A$ & $\mathrm{R} 249 \mathrm{Q}$ & 22 & nk & proximal & elbow, knee, ankle, rigid spine & $C D$ \\
\hline 8 & CE & $800 A>G$ & Y267C & 27 & spor & proximal & elbow, ankle, spine & $C D$ \\
\hline 9 & AP & $1337 \mathrm{~A}>\mathrm{T}$ & D446V & 7 & $\mathrm{nk}$ & proximal & elbow, ankle, neck & none \\
\hline 10 & Ulu & $1357 \mathrm{C}>\mathrm{T}$ & R453W & 16 & nk & none & $\begin{array}{l}\text { shoulder, hip, elbow, ankle, rigid } \\
\text { spine }\end{array}$ & none \\
\hline 11 & Kuch & $1357 \mathrm{C}>\mathrm{T}$ & R453W & 10 & fam & none & rigid spine & none \\
\hline 12 & Cat & $1357 \mathrm{C}>\mathrm{T}$ & R453W & 46 & $\mathrm{nk}$ & axial, proximal & rigid spine & $C D, P M$ \\
\hline 13 & NMC & $1583 C>G$ & T528R & 28 & $\mathrm{nk}$ & generalised & elbow, knee, ankle, rigid spine & $A, C D, P M$ \\
\hline 14 & 28 & $1583 C>G$ & T528R & 23 & spor & humeroperoneal, axial & elbow, rigid spine & $\mathrm{AF}$ \\
\hline 15 & Cal & $1622 \mathrm{G}>\mathrm{A}$ & $\mathrm{R} 541 \mathrm{H}$ & 20 & nk & humeroperoneal & rigid spine & A \\
\hline
\end{tabular}

cardiomyopathy, 25 had cardiac conduction defects or arrhythmia and 16 a combination of both.

The patient JiR had severe isolated dilated cardiomyopathy. The first symptom was dyspnea at age 24 . Echocardiography, at age 27, revealed marked left ventricular dysfunction with an ejection fraction of $22 \%$. No significant heart rhythm disturbances were detected. Thus from this point of view, the clinical phenotype of the patient is quite different from previous descriptions of heart disease in EDMD or DCM-CD as in these disorders conduction disturbances or arrhythmias occur first and left ventricular dysfunction, if present, follows. Clinical neurological examination showed no abnormalities but needle electromyography revealed mild a "myogenic" pattern in the anterior tibialis. The muscle biopsy showed mild fibre type disproportion, a non-specific alteration frequently described in EDMD. The father died of heart failure at age 55, the mother and sister were reported as healthy. None was available for analysis. JiR carried a missense heterozygous mutation causing a R435C change. The mutation affects an evolutionary highly conserved region, in exon 7 , encoding the initial portion of $C$ terminal tail of the protein and was not found among 200 chromosomes of control subjects

Patient NE5 had had a pacemaker implanted for severe conduction defects with onset at age 44 . He had no clinical signs of either skeletal muscle involvement or dilated cardiomyopathy. The parents were first degree cousins. His paternal uncle and maternal aunt both died from stroke, presumably of cardioembolic origin due to arrhythmia. The proband had two sisters and two brothers. Both sisters died suddenly at ages 25 and 32. The latter had had a pacemaker implanted and suffered from elbow contractures. One of the brothers has mildly elevated levels of CK. The other brother was diagnosed with an atrioventricular (AV) conduction defect. The proband, the maternal aunt and the brother affected with AV conduction defect were carrying the mutation $1039 \mathrm{G}>\mathrm{A}$ introducing the A347K change in a residue highly conserved in different species. The sequence change was not found among 200 chromosomes of control subjects.

\section{Unclassified muscular dystrophies}

One novel mutation (194A $>\mathrm{G}$, causing the amino acid change E65G) was identified among 32 patients presenting with unclassified muscular dystrophies (table 1). Patient NF presented with limb girdle myopathy at age 14. Later the myopathy also affected, to lesser degree, distal limb and axial muscles. The proband is now 22 years old, suffers from mild elbow contractures and no cardiac involvement has been detected so far. The father has had limb girdle muscular dystrophy since 34 years of age and at age 54 suffers from neither contractures nor cardiac disease. Although heart disease may still develop in both patients, the predominant clinical picture is that of progressive limb girdle muscular dystrophy with minor contractures and no heart involvement. The proband and his father carried the mutation that was not found among 130 chromosomes of control subjects. The mutation affects the portion of exon 1 which encodes the central rod domain.

\section{Other disorders}

No mutations were found among 23 cases of idiopathic hyperCKaemia or in two cases of lipodystrophy (table 1).

\section{DISCUSSION}

We report the mutation analysis of a large group of patients presenting a variable set of cardiomuscular phenotypes, characteristics of laminopathies, ranging from typical EDMD to isolated dilated cardiomyopathy: the inclusion criteria were more permissive than those previously established for $\mathrm{EDMD}^{23}$ due to the marked variability of clinical presentation repeatedly described in previous studies. ${ }^{18} 19$

We identified 18 mutations in the LMNA gene. No mutations were found in the X-linked STA gene in patients who were emerin-positive and did not carry mutations in $L M N A$, thus confirming the diagnostic value of the absence of emerin. The group more frequently presenting mutations was that defined as EDMD, where 15 mutations were identified, corresponding to $39.5 \%$ of the patients in the group. The analysis distinguished the patients tentatively diagnosed as EDMD into two groups, one carrying LMNA heterozygote mutations and the second presenting with wild type LMNA and STA genes. The two groups could not be distinguished based on their muscular phenotype. The clinical feature distinguishing the two groups is the cardiac involvement as 10 out of $15(66 \%)$ patients with LMNA mutations suffered from cardiac disease, while among patients not presenting LMNA/STA mutations only 5 out of $23(21 \%)$ had cardiac disorders. The mean age of the two groups is similar (21 and 26 years respectively), but, in the LMNA group, patients not presenting with cardiac involvement are the youngest (7-16 years of age, mean age 13 years) and they may still develop cardiac disorder later in life. These results confirm that cardiomyopathy is a distinctive diagnostic feature of $\mathrm{AD}$ - 
Table 3 Patients with EDMD but without LMNA mutations

\begin{tabular}{|c|c|c|c|c|c|c|}
\hline & Patient & STA analysis & Age & Distribution of myopathy & Contractures & Cardiac involvement \\
\hline 1 & FS & normal & 35 & generalised & rigid spine & sick sinus syndrome, PM \\
\hline 2 & LM & normal & 20 & humeroperoneal & elbow, ankle, rigid spine & none \\
\hline 3 & RD & normal & 18 & humeroperoneal & elbow, ankle, rigid spine & none \\
\hline 4 & Asci & normal & 38 & proximal & rigid spine & arrhythmia \\
\hline 5 & MaM & normal & 49 & proximal, distal & finger, elbow, knee, ankle, rigid spine & none \\
\hline 6 & KM & normal & 24 & proximal, distal & elbow, ankle & none \\
\hline 7 & OAr & normal & 17 & proximal, axial & elbow, ankle & conduction defect \\
\hline 8 & Kra & normal & 13 & generalised & elbow, rigid spine & none \\
\hline 9 & $\mathrm{ZPa}$ & normal & 26 & proximal & elbow, ankle & none \\
\hline 10 & Mara & normal & 12 & humeroperoneal & elbow, ankle, rigid spine & none \\
\hline 11 & NC3 & normal & 28 & proximal, distal & elbow, ankle, rigid spine & none \\
\hline 12 & NG7 & normal & 15 & proximal, distal & elbow, rigid spine & none \\
\hline 13 & NI9 & normal & 18 & proximal, distal & elbow, rigid spine & none \\
\hline 14 & NK11 & normal & 43 & proximal & elbow, rigid spine & none \\
\hline 15 & NM13 & normal & 40 & proximal, distal & elbow, rigid spine & conduction defects, PM \\
\hline 16 & NQ17 & normal & 17 & proximal, distal & elbow, rigid spine & none \\
\hline 17 & NU21 & not done & 25 & humeroperoneal & elbow, rigid spine & none \\
\hline 18 & NW22 & normal & 34 & humeroperoneal & elbow, ankle, rigid spine & none \\
\hline 19 & 191 & normal & 28 & humeroperoneal & elbow, rigid spine & none \\
\hline 20 & 263 & normal & 24 & humeroperoneal & elbow, ankle, rigid spine & none \\
\hline 21 & 686 & normal & 23 & humeroperoneal & elbow, ankle, rigid spine & none \\
\hline 22 & 2993 & normal & 25 & humeroperoneal & elbow, rigid spine & none \\
\hline 23 & DAP & not done & 37 & generalised & elbow, ankle, rigid spine & conduction defects \\
\hline
\end{tabular}

EDMD, and can be used to distinguish EDMD from other muscular disorders with muscle contractures. Some of the patients in our group may be affected with Bethlem myopathy and the genetic analysis is underway to confirm the hypothesis.

Two mutations were found among the 71 patients presenting isolated heart involvement, one among 30 cases (3.5\%) of pure idiopathic dilated cardiomyopathy and the other in 25 cases (5\%) of cardiomyopathy with conduction defects and arrhythmia. The result supports the view that the two conditions are genetically highly heterogeneous. On the other hand, since the frequency of LMNA mutations in this group of patients is similar to that of other genes responsible for cardiac disorders, our results indicate that mutations in LMNA have to be taken into account in the diagnosis of both groups of disorders.

No mutations were found among 23 cases of idiopathic hyperCKaemia, showing that the LMNA gene is not commonly involved in idiopathic hyperCKaemia.

Missense LMNA mutations are distributed along the entire LMNA gene but tend to recur within certain confined regions in exon 1, 4, 7, and 9. No genotype-phenotype correlation could be made. Moreover our study indicates that mutation spanning few amino acids of evolutionary highly conserved regions result in phenotypes with strikingly different symptoms and varying severity and point to the role of other factors in determining phenotype.

In conclusion our results show that myopathy with contractures - either isolated or associated with heart disease-is a genetically heterogeneous disease. LMNA mutations occur in almost $40 \%$ of the patients presenting muscle contractures and in approximately $70 \%$ of adult patients presenting cardiac disorders as well. They also occur in other groups of patients and in a quite unpredictable way. Finally, the identification of patients like DAP, Oar and NMI3 presenting an EDMD phenotype with cardiac conduction defects and no mutations in either LMNA or STA indicates that other genes have to be identified to fully explain the EDMD phenotype

\section{ACKNOWLEDGEMENTS}

We thank the patients and their families for agreeing to participate in this project. We thank Silvia Bione, Stavros Papadimitriou and
Marina Raffaele di Barletta, for help with the initial part of the project.

\section{Authors' affiliations}

M Vytopil, R Ricotti, D Toniolo, Institute of Molecular Genetics-CNR, Via Abbiategrasso 207, Pavia, Italy

M Vytopil, S Vohanka, Department of Neurology, University Hospital Brno, Brno, Czech Republic

J Toman, Department of Internal Medicine, St Anne's University Hospital, Brno, Czech Republic

S Benedetti, D Toniolo, DIBIT-San Raffaele Scientific Institute, Milan, Italy E Ricci, G Galluzzi, Institute of Neurology

A Dello Russo, Institute of Cardiology, Catholic University, Rome, Italy L Merlini, Neuromuscular Unit, Istituto Ortopedico Rizzoli, Bologna, Italy G Boriani, M Gallina, Institute of Cardiology, University of Bologna, Italy L Morandi, Besta Neurological Institute, Milan, Italy

L Politano, Department of Medical Genetics, University of Naples, Naples, Italy

M Moggio, L Chiveri, Department of Neurology, University of Milan, Milan, Italy

I Hausmanova-Petrusewicz, Medical Research Centre, Polish Academy of Science, Warsaw, Poland

E Ricci, Centre for Neuromuscular Diseases, UILDM-Rome Section, Rome, Italy

The research was supported by Telethon-Italy, by EU Contract EuromenMyocluster No QLG1-CT-1999-00870, and by grant IGA MZ CR NF/ 5919-3.

Correspondence to: D Toniolo, PhD, DIBIT, San Raffaele Scientific Institute, via Olgettina 58, I-20132 Milan, Italy; daniela.toniolo@hsr.it

\section{REFERENCES}

1 Onne G, et al. Mutations in the gene encoding lamin A/C cause autosomal dominant Emery-Dreifuss muscular dystrophy. Nat Genet $1999 \cdot 1 \cdot 285-8$

2 Tuurman N, Heins S, Aebi U. Nuclear lamins: their structure, assembly, and interactions. J Struct Biol 1998;122:42-66.

3 Emery AE, Dreifuss FE. Unusual type of benign X-linked muscular dystrophy. J Neurol Neurosurg Psychiatry 1966;29:338-42.

4 Muchir A, et al. Identification of mutations in the gene encoding lamins $\mathrm{A} / \mathrm{C}$ in autosomal dominant limb girdle muscular dystrophy with atrioventricular conduction disturbances (LGMDIB). Hum Mol Genet 2000:9:1453-9.

5 Van der Kooi A, et al. A newly recognized autosomal dominant limb girdle muscular dystrophy with cardiac involvement. Ann Neurol $1996 \cdot 39 \cdot 636-42$. 
6 Fatkin D, et al. Missense mutations in the rod domain of the lamin A/C gene as causes of dilated cardiomyopathy and conduction-system disease. N Engl J Med 1999;341:1715-24.

7 Shackleton $\mathbf{S}$, et al. LMNA, encoding lamin A/C, is mutated in partial lipodystrophy. Nat Genet 2000;24:153-68.

8 Speckman RA, et al. Mutational and haplotype analyses of families with familial partial lipodystrophy (Dunnigan variety) reveal recurrent missense mutations in the globular C-terminal domain of lamin A/C. Am J Hum Genet 2000;66:1192-8.

9 Cao H, Hegele RA. Nuclear lamin A/C R482Q mutation in Canadian kindreds with Dunnigan- type familial partial lipodystrophy. Hum Mol Genet 2000:9.109-12.

10 De Sandre-Giovannoli A, et al. Homozygous defects in LMNA, encoding Lamin A/C nuclear envelope proteins, cause autosomal recessive axonal neuropathy in human (Charcot-Marie-Tooth Disorder Type 2) and mouse. Am J Hum Genet 2002;70:726-36.

11 Novelli G, et al. Mandibuloacral dysplasia is caused by a mutation in LMNAencoding lamin A/C. Am J Hum Genet 2002;71:426-31.

12 Mounkes LC, Kozlov S, Hernandez L, et al. A progeroid syndrome in mice is caused by defects in A-type lamins. Nature 2003;423:298-301.

$13 \mathrm{Cao} \mathrm{H}$, Hegele RA. LMNA is mutated in Hutchinson-Gilford progeria but not in Wiedemann-Rautenstrauch progeroid syndrome. J Hum Genet 2003:48:271-4.

14 Brodsky GL, et al. Lamin A/C gene mutation associated with dilated cardiomyopathy with variable skeletal muscle involvement. Circulation 2000;101:473-6.
15 Codd MB, et al. Epidemiology of idiopathic dilated and hypertrophic cardiomyopathy. A population-based study in Olmsted County, Minnesota, 1975-1984. Circulation 1989;80:564-72.

16 Relle A, et al. Retrospective study of a large population of patients with asymptomatic or minimally symptomatic raised serum creatine kinase levels. J Neurol 2002;249:305-11.

17 Vytopil M, et al. Frequent low penetrance mutations in the Lamin A/C gene, causing Emery Dreifuss muscular dystrophy. Neuromuscul Disord 2002;12:958-63.

18 Bonne G, et al. Clinical and molecular genetic spectrum of autosomal dominant Emery- Dreifuss muscular dystrophy due to mutations of the lamin A/C gene. Ann Neurol 2000;48:170-80.

19 Brown CA, et al. Novel and recurrent mutations in lamin A/C in patients with Emery- Dreifuss muscular dystrophy. Am J Med Genet 2001;102(4):359-67.

20 Raffaele Di Barletta $M$, et al. Different mutations in the LMNA gene cause autosomal dominant and autosomal recessive Emery-Dreifuss muscular dystrophy. Am J Hum Genet 2000;66:1407-12.

21 Maguire A, et al. X-linked cerebellar ataxia and sideroblastic anaemia associated with a missense mutation in the $A B C 7$ gene predicting $V 411 \mathrm{~L}$. Br J Haematol 2001;115:910-7.

22 Flavell DM, et al. Peroxisome proliferator-activated receptor alpha gene variants influence progression of coronary atherosclerosis and risk of coronary artery disease. Circulation 2002;105:1440-5.

23 Yates JR. 43rd ENMC International workshop on Emery-Dreifuss muscular dystrophy, 22 June 1996, Naarden, the Netherlands. Neuromuscul Disord 1997:7:67-9. 